\section{Röntgen-Plaketten 2020 und 2021 verliehen}

Gleich 2 Röntgen-Plaketten hat die Stadt Remscheid am 18. September 2021 an renommierte Wissenschaftler verliehen - an Professor Wolfgang Schlegel, Pionier der Medizinischen Physik, und an den Astrophysiker Günther Hasinger. In diesem Jahr wurden 2 Plaketten verliehen, da die Verleihung an Professor Günther Hasinger im Jahr 2020 pandemiebedingt ausfallen musste.

Die Preisträger der in Fachkreisen sehr begehrten Röntgen-Plakette sind Professor Wolfgang Schlegel, Pionier der Medizinischen Physik (Preisträger 2021), und der Astrophysiker Professor Günther Hasinger von der ESP Madrid (Preisträger 2020). Die feierliche Verleihung der Plaketten fand am 18. September in der Aula des RöntgenGymnasiums in Remscheid durch den Oberbürgermeister der Stadt, Burkhard Mast-Weisz, statt. Im Anschluss lud das Deutsche Röntgen-Museum zu einem kleinen Empfang ins Foyer des Museums ein. In diesem Jahr wurden 2 Plaketten verliehen, da die Verleihung an Professor Günther Hasinger im vergangenen Jahr pandemiebedingt ausfallen musste.

Professor Wolfgang Schlegel ist ein Pionier der Medizinischen Physik. Zahlreiche Verfahren und Techniken, die heute in Kliniken rund um den Globus eine präzise Bestrahlung von Tumoren ermöglichen, basieren

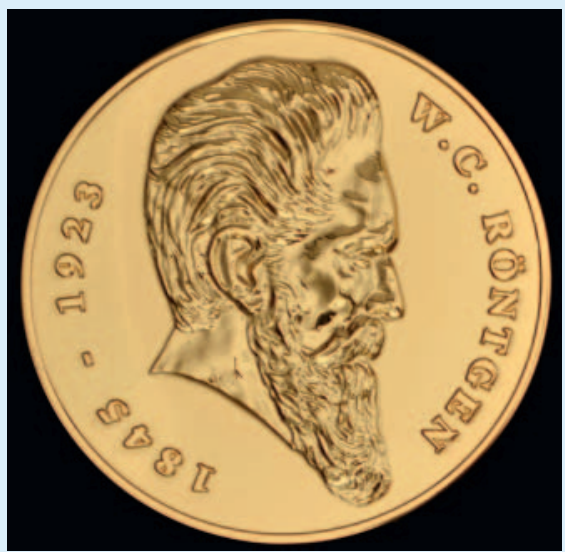

Die begehrte Röntgen-Plakette. (c) Deutsches Röntgen-Museum

auf den Entwicklungen Schlegels und seiner Mitarbeiterinnen und Mitarbeiter. Zahllose Patientinnen und Patienten weltweit haben es seinen Erfindungen zu verdanken, dass ihre Tumoren präzise und schonend bestrahlt und ihre Heilungschancen so verbessert werden konnten. Mit der RöntgenPlakette würdigte die Stadt Remscheid Professor Schlegels herausragende Leistungen zur Entwicklung der intensitätsmodulierten konformalen Strahlentherapie.

Der Astrophysiker Professor Günther Hasinger wurde mit der Röntgen-Plakette für seine herausragenden Forschungen auf dem Gebiet der Röntgenastronomie gewürdigt. In den letzten Jahrzehnten hat sich Günther
Hasinger mit der Erforschung des Röntgenhintergrunds beschäftigt. Seine Untersuchungen halfen nachzuweisen, dass diese diffuse Röntgenstrahlung hauptsächlich von unzähligen Schwarzen Löchern in aktiven Galaxienzentren stammt, die im Laufe der kosmischen Geschichte wachsen und strahlen. Aktuell befasst er sich mit den schwachen Fluktuationen im kosmischen Röntgenund Infrarot-Hintergrund, die auf die Existenz einer sehr frühen Population Schwarzer Löcher hinweisen, und untersucht die Frage, ob es sich dabei möglicherweise um die bisher unverstandene Dunkle Materie handelt.

Die Röntgen-Plakette gibt es seit 1951 in Anlehnung an den 50. Jahrestag der Verleihung des ersten Nobelpreises an Wilhelm Conrad Röntgen. Die Plakette wird vom Oberbürgermeister oder der Oberbürgermeisterin der Stadt Remscheid an Persönlichkeiten verliehen, die sich im weitesten Sinne um den Fortschritt und die Verbreitung von Röntgens Entdeckungen besonders verdient gemacht haben. Sie ist eine in Fachkreisen hoch angesehene und begehrte wissenschaftliche Auszeichnung geworden. Die Gesellschaft der Freunde und Förderer des Deutschen Röntgen-Museums Remscheid-Lennep e. V. wählt geeignete Wissenschaftlerinnen und Wissenschaftler aus aller Welt aus und schlägt sie dem Oberbürgermeister oder der Oberbürgermeisterin zur Ehrung vor. 